\title{
Subjective Well-being Effects for Electric Power Universal Service Recipients in China from a Culture Poverty Perspective
}

\author{
Hui-ru Zhao, Hui-juan Huo \\ School of Economics and Management, North China Electric Power University, Beijing, China
}

Email address:

315159099@qq.com (Hui-juan Huo)

To cite this article:

Hui-ru Zhao, Hui-juan Huo. Subjective Well-being Effects for Electric Power Universal Service Recipients in China from a Culture Poverty Perspective. International Journal of Energy and Power Engineering. Vol. 4, No. 1, 2015, pp. 32-38. doi: 10.11648/j.ijepe.20150401.15

\begin{abstract}
Lack of cultural knowledge, poor livelihood and inappropriate value orientation of electric power universal service recipient groups basically influence their electricity consumption habit in china. In order to assess the effect of power universal service distribution and measure the welfare of recipient groups, this paper researches individual electricity consumption mentality and behavior through behavioral economics theory, establishes a subjective welfare function tracing recipient individual based on cumulative prospect theory, constructs the recipient groups' subjective welfare function following the traditional social welfare function and researches the influence of the changes of psychological reference points. Through example analysis combined with function feasibility, this paper proves the subjective well-being effect of recipient groups declines when the psychological reference point increases.
\end{abstract}

Keywords: Electric Power Universal Service, Consumer Psychology, Subjective Well-Being, Cumulative Prospect Theory

\section{Introduction}

Electric Power Universal Service (EPUS) is a government funded program, aiming to insure people who live in remote areas can consume electric power at affordable prices to improve their welfare level in China. When assessing the effect of EPUS, we take account into the recipient groups ' social welfare. Most EPUS receipts live in remote areas with little literal knowledge, poor livelihood habits, backward value orientations and old style customs compared with current leading culture of our society in China. This unique environment can affect consumer psychology and electrical behavior of recipient individual, thus influence the EPUS recipient groups' welfare level. Based a social welfare perspective, the paper will research the EPUS recipient groups' welfare level for Chinese government to assess the effect of EPUS.

In order to measure social welfare level, most international and domestic scholars are focusing their research on assessing the welfare level through analyzing objective factors including income and expenditure based on an individual economic consumer hypothesis. Ref. [1] has explained the designed social welfare functions as a function reflecting the interaction of min income level, average income level and Gini coefficient. Ref. [2] set up a comprehensive evaluation of the social welfare system tracing economic growth and inequality distribution through change the income-based individual utility function and social welfare function into standard satisfaction function. Ref. [3] established a social welfare maximization condition function through introducing currency measurement into the social welfare function. Ref. [4] created a social welfare evaluation model measuring mix indexes including income, expenditure and health level amid functions and capabilities space. Ref. [5] set up an energy service social welfare function analyzing income and expenditure. Ref. [6] established a social welfare evaluation model using axiomatic system and defining random individual situation based on preference theory. Ref. [7] analyzed unique problems of China's social welfare through summarizing domestic economic and social welfare evolution changed with political and economic development process. Ref. [8] measured income-based social welfare level in short and long term through introducing equal opportunity rule into traditional welfare functions.

Base on social people hypothesis, value orientation, education level and other subjective factors can influence social welfare level through interacting with consumer psychology and behaviors. Ref. [9] researched China's individual consumer behavior and utility level applying 
mental accounting theory, prospect theory and consumer behavior analysis. Ref. [10] established a consumer habits-based optimal consumption - saving model through introducing the loss aversion model of consumption habits theory and prospect theory into the consumption and saving situation. Ref. [11] researched the influence of psychological factors on the energy consumption behavior of Chinese urban residents by explaining examples in Shenyang and Dalian City. Ref [12] built the evaluation model from region economy etc.to measure the power universal service economic and non-economic welfares based on the social welfare function theory and prospect theory.

EPUS recipients' welfare can be influenced by subjective factors (e.g. educational level) according to the consumption economics theory. The recipient individuals with different educational level have different cognitive level for electricity, which causes the different individual welfare. The Subjective Well-being (SWB) is defined as the welfare induction, which recipient individual gets from the electricity under the influence of subjective factors (e.g. educational level). SWB embodies the satisfaction for improving their livelihood and beautifying their future life under the psychological effects. This literature from the angle of cultural poverty, focus on the subjective welfare resulting from the educational level differences and assumes that other subjective factors have no difference.

The method we propose consists of three steps. In the first step, one has to explain the characteristics of cultural poverty in the EPUS recipient and to put forward the frugalness and herd mentality as their consumer psychological characteristics. In the second step, according to herd consumer behavior and thrifty consumption behavior, one constructs the SWB model to measure the EPUS individual SWB based on the cumulative prospect theory (CPT). The third step involves the SWB measurement of the EPUS recipient groups of different educational level based on the welfare economics.

\section{EPUS Recipients' Cultural Poverty and Consumer Psychology in China}

Culture poverty refers to a group or an individual lives with less literal knowledge, poorer livelihood habits, backward value orientations and older style customs compared with current leading culture of our society, which will hamper their willingness to improve livelihood and spiritual needs. In china, most EPUS recipients are peasant households living in remote areas, with little access to instant information, backward mode of production and the concept of rigid. People aimed culture poverty usually poorly educated and lack of scientific knowledge, poor livelihood conditions lead to outdated value orientations, old-fashioned thinking, low moral standards and weak sense of democracy in China. Influenced by culture poverty, EPUS recipients lead a life of nature, thrifty and emphasis on tribalism, which forms their consuming psychology with features including.

(1) Choosing substantial and low-priced commodities

People living in culture poverty always buy substantial and cheap commodities to satisfy basic needs at affordable prices in China. In terms of home appliances purchase, recipient individuals tend to choose essential commodities such as CRT television (TV), washing machine and small-sized electric appliances rather than new products including LCD $\mathrm{TV}$, air conditioner and computers.

(2) Extreme frugalness

People living in culture poverty always lead an extreme frugal live with thrifty mentality to save money through eating ordinary food, using common daily consumer goods in China. They tend to cut lighting time and reduce the frequency of home appliance to save electricity fees.

(3) Herd mentality

EPUS recipients under culture poverty have limited access to outward information due to outdated public transportation and telecommunications infrastructure in China. Limited local information acquisition and collectivism in big families lead recipient individuals to choose similar products as other people already bought.

\section{EPUS Recipient Individual Subjective Well-being Model Based on Cumulative Prospect Theory}

\subsection{Cumulative Prospect Theory}

Cumulative prospect theory (CPT) is resulted from connecting cognitive psychology and behavioral science with rich experimental verification. CPT reflects decision made the procedures in uncertain conditions, which is proper to measure the bounded rationality of EPUS recipients. The recipient individual psychological reference points and value function can reflect individual subjective well-being degree. This paper refers CPT analysis framework to track the subjective well-being model of recipient individual.

Psychological reference point reflects the expectation of electricity while value orientation function tracks the value an individual receives during electricity. Based on cumulative prospect theory, the value loss function is shown as follows:

$$
v^{-}(\Delta x)=-\lambda\left(a q^{*}-a q\right)^{\beta}, q<q^{*}
$$

And the value profit function is shown as follows:

$$
v^{+}(\Delta x)=\left(a q-a q^{*}\right)^{\alpha}, q \geq q^{*}
$$

Where $\alpha$ and $\beta$ represent the risk attitude coefficient; $\lambda$ represents the avoid coefficient of value loss. According to Tvesky and Kahneman's research experience, $\alpha$ and $\beta$ are equal to $0.88, \lambda$ is equal to 2.25 .

Base on cumulative prospect theory, the recipient individual undergoes two stages of subjective well-being effect. The first stage is to determine the psychological reference point as $T$, which the recipient individual expects to receive from electricity. The second stage is to set a recipient individual expected value, which embodies the distance between the individual subjective well-being and the psychological reference point. The 
psychological reference point and expected value contribute a recipient individual subjective well-being.

\subsection{The Recipient Individual SWB Influenced by Herd Consumer Behavior}

Influenced by herd consumer behavior, the EPUS recipient individual subjective well-being will increase with the growth of actual electricity, and be known as the marginal increment for utility. This paper assumes the marginal increment carries linear features. The subjective well-being effect of a recipient individual under herd consumer behavior is shown as follows:

$$
\mathrm{U}_{z}=T_{z}\left(q^{*}\right)+V_{z}\left(\Delta x_{\mathrm{z}}\right)
$$

Where $q^{*}$ represents the reference electricity usage in line with psychological reference point; $\mathrm{T}_{\mathrm{z}}\left(q^{*}\right)=a q^{*}$ represents the psychological reference point utility influenced by herd consumer behavior; $V_{\mathrm{z}}\left(\Delta x_{\mathrm{z}}\right)$ represents the expected value function influenced by herd consumer behavior; $\Delta x_{z}=a\left(q-q^{*}\right)$ represents the cognitive profit and loss from actual electricity; $a$ represents the coordinate coefficient, $a>0$; $q$ represents the recipient individual actual electricity.

Influenced by herd consumption habit, if $q \leq q^{*}$, the individual psychological demand to follow major groups will be not satisfied, as follows:

$$
\Delta x_{z}=a\left(q-q^{*}\right) \leq 0
$$

If $q \geq q^{*}$, the psychological demand to follow major groups will be satisfied, as follows:

$$
\Delta x_{z}=a\left(q-q^{*}\right)>0
$$

Based on CPT, the recipient individual expected value function is calculated as follows:

$$
V\left(\Delta x_{z}\right)= \begin{cases}\int_{\Delta x_{z}^{\min }}^{\Delta x_{z}} v^{-}\left(\Delta x_{z}\right) d F\left(\Delta x_{z}\right) & \Delta x_{z}<0 \\ \int_{\Delta x_{z}^{\min }}^{\Delta x_{z}} v^{+}\left(\Delta x_{z}\right) d F\left(\Delta x_{z}\right) & \Delta x_{z} \geq 0\end{cases}
$$

where $\mathrm{F}\left(\Delta \mathrm{x}_{\mathrm{z}}\right)$ represents the recipient individual cognitive profit and loss distribution function influenced by herd consumer behavior, which is uniform distribution or normal distribution, $\Delta x_{z}^{\min }=-a q^{*}$.

So the recipient individual subjective well-being function is calculated as follows,

$$
\mathrm{U}_{z}=\left\{\begin{array}{cc}
a q^{*}-\int_{0}^{q} \lambda_{z}\left(a q^{*}-a q\right)^{\beta_{z}} d F(q) & q \leq q^{*} \\
a q^{*}+\int_{0}^{q}\left(a q-a q^{*}\right)^{\alpha_{z}} d F(q) & q>q^{*}
\end{array}\right.
$$

\subsection{The Recipient Individual SWB Influenced by Thrifty Consumer Behavior}

Thrifty consumer behavior is also a psychological factor. When the actual electricity and electricity fees increase, the recipient individual subjective well-being utility will decrease, in line with the diminishing marginal utility.
Assuming the diminishing marginal utility function carry linearity, the subjective well-being function influenced by thrifty consumer behaviors is as follows:

$$
\mathrm{U}_{r}=T_{r}\left(q^{*}\right)+V_{r}\left(\Delta x_{r}\right)
$$

Where $T_{r}\left(q^{*}\right)=b\left(I^{e}-p q^{*}\right)$ represents the mental reference point utility value of the recipient individual with thrifty consumer behavior; $V_{r}\left(\Delta x_{r}\right)$ represents the value function influenced by thrifty consumer behavior; $\Delta x_{r}=b\left(p q^{*}-p q\right)$ represents the cognitive profit and loss from actual electricity; $b$ represents the coordinate coefficient, $b>0 ; I^{\mathrm{e}}$ represents the maximum electric fee expenditure; $p$ is electric price.

If $q \leq q^{*}$, the recipient individual frugal consumer psychology and inexpensive consumer psychology will be satisfied, as follows:

$$
\Delta x_{r}=b\left(p q^{*}-p q\right) \geq 0
$$

If $q \geq q^{*}$, the recipient individual frugal consumer psychology and inexpensive consumer psychology will not be satisfied, he will feel loss, as follows:

$$
\Delta x_{r}=b\left(p q^{*}-p q\right)<0
$$

Influenced by thrifty consumer behavior, the recipient individual expected value function is as follows:

$$
V_{r}\left(\Delta x_{r}\right)= \begin{cases}\int_{\Delta x_{r}^{\min }}^{\Delta x_{r}} v^{+}\left(\Delta x_{r}\right) d F\left(\Delta x_{r}\right) & \Delta x_{r} \geq 0 \\ \int_{\Delta x_{r}^{\min }}^{\Delta_{r}} v^{-}\left(\Delta x_{r}\right) d F\left(\Delta x_{r}\right) & \Delta x_{r}<0\end{cases}
$$

Where $\mathrm{F}\left(\Delta \mathrm{x}_{\mathrm{r}}\right)$ represents the cognitive profit and loss influenced by thrifty consumer behavior, which is uniform distribution or normal distribution, $\Delta x_{r}^{\min }=b p q^{*}-b p q^{\max }$, $\mathrm{q}^{\max }$ is the maximum electricity usage under EPUS.

So the recipient individual subjective well-being function influenced by thrifty consumer behavior is as follows:

$$
\mathrm{U}_{r}=\left\{\begin{array}{cc}
b\left(I^{e}-p q^{*}\right)+\int_{q}^{q_{\max }}\left(b p q^{*}-b p q\right)^{\alpha_{r}} d F(q) & q \leq q^{*} \\
b\left(I^{e}-p q^{*}\right)-\int_{q}^{q_{\max }} \lambda_{r}\left(b p q-b p q^{*}\right)^{\beta_{r}} d F(q) & q>q^{*}
\end{array}\right.
$$

\subsection{The Recipient Individual SWB Function}

After analyzing the welfare effect influenced by herd consumer behavior and thrifty consumer behavior, this paper explains the recipient individual SWB function is as follows:

$$
w_{i}=U_{z}+U_{r}
$$

Where $w_{i}$ represents the SWB of recipient individual $i$.

According to formula (7) and (13), the recipient individual subjective well-being can be calculated.

\section{EPUS Recipient Groups SWB Model}

In order to the recipient groups' subjective well-being 
model in China, this paper analyzes the psychological reference point, sets up individual well-being model based on traditional social welfare function on the premise of individual welfare comparability and calculates the welfare effects when the psychological reference point changes.

\subsection{The Recipients' Psychological Reference Point}

According to the psychological reference point, under current cognitive level of EPUS recipients in china, the recipient individual should obtain the enough utility $T$ after consuming the reference electricity usage $q^{*}$. The psychological reference point $\mathrm{T}$ and the recipient individual subjective well-being $w_{i}$ depend on the reference electricity usage $q^{*}$.

Under culture poverty, different recipient individual has different judgments on the reference electricity usage. This paper assumes that the judgment about the reference electricity usage is only affected by their educational level. The better education an individual gets, he will be more willing to use more home appliances to improve his living conditions, then his reference electricity usage is larger, otherwise the reference electricity usage is small if an individual receives little education.

The recipients' educational level can be divided into three levels in Chinese EPUS region, namely the level of primary education, junior middle school and high school level. So the reference electricity usage is shown as follows:

$$
q_{j}^{*}=\delta_{j} \times q_{0}^{*}
$$

Where $q_{j}^{*}$ represents the recipient individual reference electricity usage of the $j$ education level; $q_{0}^{*}$ represents the benchmark reference electricity usage; $\delta_{j}$ represents the coordinate coefficient of electricity usage of the $j$ education level.

$$
\text { Set } q_{0}^{*}=q_{3}^{*} \text {, so }
$$

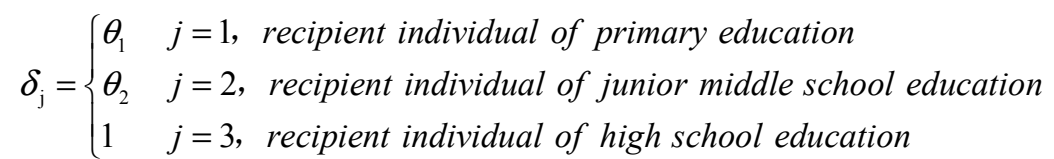

Where $\theta_{1}$ represents the electricity usage coordinate coefficient of recipient individual of primary education; $\theta_{2}$ represents the electricity usage coordinate coefficient of recipient individual of junior middle school education; $\theta_{1}<\theta_{2}<1$.

The psychological reference point for recipient individual is shown as follows:

$$
T_{j}=T_{z j}\left(q^{*}\right)+T_{r j}\left(q^{*}\right)
$$

Where $T_{j}$ represents the psychological reference point utility of the $j$ education level recipient individual; $T_{z j}\left(q^{*}\right)$ represents the psychological reference point utility influenced by herd consumer behavior; $T_{r j}\left(q^{*}\right)$ represents the psychological reference point utility influenced by thrifty consumer behavior. Then the Equation (15) can be shown:

$$
T_{j}=a q_{j}^{*}+b\left(I^{e}-p q_{j}^{*}\right)
$$

\subsection{The SWB Function for EPUS Recipient Groups}

According to the theory of traditional social welfare, social welfare is the sum of all individual well-being when an individual well-being is countable. As for EPUS, based on cumulative prospect theory, this paper establishes a model to make individual well-being countable. Meanwhile, assuming all personal information except education level are the same, so individual well-being effect is comparable. On this basis, this paper sets up the social welfare function $\mathrm{W}$ for EPUS through applying of traditional social welfare function.

Supposing there are $\mathrm{M}$ individuals in an EPUS region, then social welfare function $\mathrm{W}$ can be expressed as:

$$
W=\sum_{i=1}^{M} w_{i}
$$

Where $w_{i}$ represents the SWB function for individual $i$.

\subsection{Effect of the Reference Point Changes on SWB}

The difference between reference electricity usage and actual electricity usage influences individual subjective well-being effect. The subjective well-being effect changes along with the reference electricity usage changes.

With the deep implementation of EPUS, recipients under culture poverty change their cognitive level, consumer psychology and consumer behavior, then reference electricity usage and psychological reference point change. According to Bell's theory about spending habits, it presumes that EPUS recipients' psychological reference point and reference electricity usage will increase along the growth of actual electricity usage. Therefore, based on the theory of consumer habits, the reference electricity usage of a recipient individual $i$ during $\mathrm{t}+1$ period is as follows:

$$
q_{i(t+1)}^{*}=\left\{\begin{array}{cl}
(1-\varphi) q_{i t}^{*}+\varphi q_{i t} & q_{i t} \geq q_{i t}^{*} \\
q_{i t}^{*} & q_{i t}<q_{i t}^{*}
\end{array}\right.
$$

where $q_{i t}^{*}$ represents reference electricity usage of recipient individual $i$ during $t$ period, $q_{i t}$ represents individual $i$ actual electricity usage during $t$ period; $\varphi$ represents the continued strength of consumer habit. If $\varphi=0$, the reference electricity usage during $t+1$ period is equal to the reference electricity usage during $t$ period; If $\varphi=1$, the reference electricity usage 
during $t+1$ period is equal to the actual electricity usage during $t$ period.

The psychological reference point $T_{\text {it }}$ of a recipient individual $i$ during $t$ period is as follows:

$$
T_{i t}=a q_{i t}^{*}+b\left(I^{e}-p q_{i t}^{*}\right)
$$

Then the psychological reference point $\mathrm{T}_{\mathrm{i}(\mathrm{t}+1)}$ of a recipient individual $i$ during $t+1$ period is as follows:

$$
\begin{aligned}
& W_{t}=\sum_{i=1}^{M}\left\{\rho\left[a q_{i t}^{*}-\int_{0}^{q} \lambda_{z}\left(a q_{i t}^{*}-a q_{i}\right)^{\beta_{z}} d F\left(q_{i}\right)+b\left(I^{e}-p q_{i t}^{*}\right)+\int_{q}^{q_{\max }}\left(b p q_{i t}^{*}-b p q_{i}\right)^{\alpha_{r}} d F\left(q_{i}\right)\right]\right. \\
& \left.+(1-\rho)\left[a q_{i t}^{*}+\int_{0}^{q}\left(a q-a q_{i t}^{*}\right)^{\alpha_{z}} d F\left(q_{i}\right)+b\left(I^{e}-p q_{i t}^{*}\right)-\int_{q}^{q_{\max }} \lambda_{r}\left(b p q-b p q_{i t}^{*}\right)^{\beta_{r}} d F\left(q_{i}\right)\right]\right\}
\end{aligned}
$$

To research the influence of psychological reference point changes on subjective well-being effect, this paper assumes that all individual actual electricity usages and maximum electric fee expenditures are fixed. When compare the individual subjective well-being effect during $t$ and $t+1$ period, we can get the subjective well-being change function as follows:

$$
T_{i(t+1)}=\left\{\begin{array}{cc}
(1-\varphi) T_{i t}+a q_{i t}+b\left(I^{e}-p q_{i t}\right) & q_{i t} \geq q_{i t}^{*} \\
T_{i t} & q_{i t}<q_{i t}^{*}
\end{array}\right.
$$

Therefore when the psychological reference point changes, the subjective welfare effect for EPUS recipient group during $\mathrm{t}$ period is as follows:

$$
\Delta w_{i}=w_{i(t+1)}-w_{i t}
$$

If $q_{i} \geq q_{i t}^{*}$, the recipient individual tends to improve electricity usage in next time period following consumers habit, so $q_{i} \geq q_{i(t+1)}^{*} \geq q_{i t}^{*}$, the recipient individual subjective well-being change function is as follows:

$$
\begin{aligned}
\Delta w_{i} & =a \varphi\left(q_{i}-q_{i t}^{*}\right)+\int_{0}^{q}\left(1-(1-\varphi)^{1+\alpha_{z}}\right) a^{\alpha_{z}}\left(q_{i}-q_{i t}^{*}\right)^{\alpha_{z}} d F\left(q_{i}\right) \\
& +b \varphi p\left(q_{i t}^{*}-q_{i}\right)-\int_{q}^{q_{\max }} \lambda_{r}\left(1-(1-\varphi)^{1+\beta_{r}}\right) b^{\beta_{r}} p^{\beta_{r}}\left(q_{i t}^{*}-q_{i}\right)^{\beta_{r}} d F\left(q_{i}\right)
\end{aligned}
$$

If $q_{i}<q_{i}^{*}$, the recipient individual remains electricity usage in next time period. so $q_{i}<q_{i(t+1)}^{*}=q_{i t}^{*}$, there is no apparent change in individual subjective well-being.

Based on the above, $M$ individual SWB effect changes

$$
\begin{gathered}
\Delta W=\sum_{i=1}^{N}\left\{a \varphi\left(q_{i}-q_{i t}^{*}\right)+\int_{0}^{q}\left[1-(1-\varphi)^{1+\alpha_{z}}\right] a^{\alpha_{z}}\left(q_{i}-q_{i t}^{*}\right)^{\alpha_{z}} d F\left(q_{i}\right)+\right. \\
\left.\quad b \varphi p\left(q_{i t}^{*}-q_{i}\right)-\int_{q}^{q_{\max }} \lambda_{r}\left[1-(1-\varphi)^{1+\beta_{r}}\right] b^{\beta_{r}} p^{\beta_{r}}\left(q_{i t}^{*}-q_{i}\right)^{\beta_{r}} d F\left(q_{i}\right)\right\}
\end{gathered}
$$

\section{Example Analysis}

This paper takes a certain EPUS region in China as the example to focus on research of EPUS subjective well-being and analyze the influence of psychological reference point changes on social welfare.
The EPUS region is remote with poor economics and livelihood, insufficient power grid infrastructures and culture poverty. There are 30 peasant households with difference income, education and power consumption level (Chart 1). Electric fee is set at $0.5 \mathrm{RMB} / \mathrm{kWh}$.

Chart 1. Example details

\begin{tabular}{llll}
\hline Index & & Number of peasant households & Ration (\%) \\
\hline & RMB 1,500 2,000 & 3 & 10.0 \\
Average annual income & RMB 2,000 2,500 & 9 & 30.0 \\
& RMB 2,500 3,000 & 13 & 43.3 \\
& More than RMB 3,000 & 5 & 16.7 \\
Education degree & primary school & 2 & 6.7 \\
& Junior middle school & 22 & 73.3 \\
high school & 6 & 3 & 20.0 \\
Annual power usage & $300 \sim 400 \mathrm{kWh}$ & 12 & 10.0 \\
& $400 \sim 500 \mathrm{kWh}$ & 13 & 40.0 \\
& $500 \sim 600 \mathrm{kWh}$ & 2 & 43.3 \\
\end{tabular}


The recipient peasant households are influenced by frugal and herd behaviors when using electricity, the cognitive profit and loss from actual electricity are shown as:

$$
\begin{gathered}
\left\{\begin{array}{l}
\Delta x_{z}=q-q^{*} \\
\Delta x_{r}=0.5\left(q^{*}-q\right)
\end{array}\right. \\
q^{*}= \begin{cases}400 & \text { recipient peasant household of primary education } \\
500 & \text { recipient peasant households of junior middle school education } \\
550 & \text { recipient peasant households of high school education }\end{cases}
\end{gathered}
$$

The recipient peasant households choose reference electricity usage concerned with education degree only. Peasants with difference education degrees choose difference
Assuming that the provided maximum power volume for the recipients is $1,000 \mathrm{kWh} /$ year and the peasant cognitive profit and loss follows uniform distribution, as follows:

$$
F_{z}(q)=F_{r}(q)=\frac{q}{1000}
$$

\begin{tabular}{|c|c|c|c|c|}
\hline No. & $\begin{array}{l}\text { Education } \\
\text { degree }\end{array}$ & $\begin{array}{l}\text { Reference } \\
\text { power usage } \\
\text { (kWh) }\end{array}$ & $\begin{array}{l}\text { SWB } \\
\text { during } t \\
\text { (unit) }\end{array}$ & $\begin{array}{l}\text { SWB } \\
\text { during } t+1 \\
\text { (unit) }\end{array}$ \\
\hline 1 & primary & 400 & 339 & 339 \\
\hline 2 & primary & 500 & 490 & 490 \\
\hline 3 & Junior high & 400 & 411 & 411 \\
\hline 4 & Junior high & 500 & 409 & 409 \\
\hline 5 & Junior high & 500 & 561 & 561 \\
\hline 6 & Junior high & 500 & 585 & 585 \\
\hline 7 & Junior high & 500 & 409 & 409 \\
\hline 8 & Junior high & 500 & 355 & 355 \\
\hline 9 & Junior high & 500 & 618 & 618 \\
\hline 10 & Junior high & 500 & 561 & 561 \\
\hline 11 & Junior high & 500 & 637 & 637 \\
\hline 12 & Junior high & 500 & 661 & 661 \\
\hline 13 & Junior high & 500 & 774 & 774 \\
\hline 14 & Junior high & 500 & 436 & 436 \\
\hline 15 & Junior high & 500 & 673 & 673 \\
\hline 16 & Junior high & 500 & 3003 & 3003 \\
\hline 17 & Junior high & 500 & 3042 & 3042 \\
\hline 18 & Junior high & 500 & 3111 & 3111 \\
\hline 19 & Junior high & 500 & 3001 & 3001 \\
\hline 20 & Junior high & 500 & 3014 & 3014 \\
\hline 21 & Junior high & 500 & 3030 & 3030 \\
\hline 22 & Junior high & 500 & 3726 & 3726 \\
\hline 23 & Junior high & 500 & 3625 & 3625 \\
\hline 24 & Junior high & 500 & 3923 & 3923 \\
\hline 25 & High school & 550 & 487 & 487 \\
\hline 26 & High school & 550 & 781 & 781 \\
\hline 27 & High school & 550 & 3051 & 3051 \\
\hline 28 & High school & 550 & 3060 & 3060 \\
\hline 29 & High school & 550 & 3647 & 3647 \\
\hline 30 & High school & 550 & 3884 & 3884 \\
\hline total & & & 54684 & 52304 \\
\hline
\end{tabular}

So the SWB level for the recipient group during $\mathrm{t}$ period is calculated to be 54,684 units as Chart 2 .

Chart 2. Subjective well-being for the EPUS region
The recipient peasant household tends to change reference electricity usage according to the theory of consumption habit. Assuming the actual electricity usage and the maximum electric fee expenditure are fixed during $t+1$ period, the continued strength of consumer habit $\varphi$ is set at 0.5 , then recipient group subjective well-being utility is changed to 52,304 units, falling 2,380 units from $t$ period. The calculations are as Chart 2.

If the actual electricity usage and electric fee expenditure are fixed during the next period, the recipients' SWB effect will fall when the reference electricity usage increases in EPUS regions.

\section{Conclusion}

EPUS recipients usually carry the characteristic of culture poverty in China. From culture poverty perspective, EPUS recipient individuals act herd consumer behavior and thrifty consumer behavior. Based on the two kinds of behaviors, this literature introduces the psychological reference point and value function from CPT to establish the individual SWB function. It described a psychological reference point based on the differences of educational degree and established the groups' SWB function on the premise of countable individual welfare indexes, and analyzes the influence of psychological reference point changes on individual SWB effect. With empirical analysis to verify the feasibility of the function, this paper at last proved that the recipient SWB effect declined when the psychological reference point increases. Thus, Chinese government authorities should pay attention to emphasizing equipment maintenance and improving power service standards for enjoying the electricity and enhancing the SWB at proper time.

This paper not only provides a quantification method to measure SWB effect, but also introduces a theoretical basis to assess EPUS implementation effect comprehensively, including subjective factors.

\section{Acknowledgements}

This study is supported by The National Natural Science Foundation of China (71373076). 


\section{References}

[1] Zhao Zhijun. Optimization of Income Distribution and Social Welfare Function [J]. The Journal of Quantitative \& Technical Economics, 2011, 09: 61-74.

[2] Ou Yangkui. Comprehensive Measure of Economic Growth and Inequality Satisfaction Entropy and Social Welfare [J]. Modern Economic Science, 2010, 05:13-22+124.

[3] Meng Qingchun, Wang Jianmin. A Research on Social Welfare Maximization and Resource Expenditure Minimization Considering the Money Metric Social Welfare Function [J]. Chinese Journal of Management Science, 2001, 06:22-26.

[4] Yang Aiting, Song Deyong. The Measurement of Chinese Social Welfare and the Analysis of Low-welfare Growth [J]. The Journal of Quantitative \& Technical Economics, 2012, $11: 3-17+148$

[5] Holger Schlör. Measuring social welfare, energy and inequality in Germany [J]. Applied Energy, 2012, 97:135-142.

[6] Marcus Pivato. Social welfare with incomplete ordinal interpersonal comparisons [J]. Journal of Mathematical Economics, 2013, 49:405-417.
[7] Koen, Rutten. Social Welfare in China: The role of equity in the transition from egalitarianism to capitalis [EB/OL]. http://hdl.handle.net/10398/8018, 2010-03-04

[8] Rolf Aaberge, Magne Mogstad, Vito Peragine. Measuring long-term inequality of opportunity [J].Journal of Public Economics.2011,95:193-204

[9] Li Xiaojing. Analysis for Chinese Consumers Behavior from the Perspective of Behavioral Economics [D]. Hebei University of Economics and Business, 2012.

[10] Zhao Guoqin. Study on Consumption-Savings and Retirement Based-on Prospect Theory [D]. Tianjin University, 2008.

[11] Sun Yan,Jiang Ling. Study on Psychological Factors on the Influence of Urban Energy Consumption behavior[J]. Consumer Economics, 2013, 03: 77-79+95.

[12] Zhao Huiru, Guo Sen, Zhang Qi, Li Chunjie. Social Welfare Evaluation of Electric Universal Service in China: From the Perspective of Sustainability [J]. Sustainability, 2014,6(8):4949-1965.

[13] Lou Linli. Theoretical and Empirical Research on the Economics of Subjective Well-being [D]. Fudan University, 2009. 